Revista Chilena de Antropología $N^{\circ} 5,1986,103-120$

Facultad de Filosofía, Humanidades y Educación

Universidad de Chile, Santiago, Chile

\title{
Los Salineros en la costa de Chile Central*
}

\author{
Daniel Quiroz, Patricio Poblete y \\ Juan C. Olivares
}

\section{INTRODUCCIÓN}

Durante el período 1980-1982, y dentro del marco de una investigación interdisciplinaria sobre problemas de extrema pobreza, pudimos estudiar, en particular, un modo de vida, en el cual se establece una compleja red de relaciones entre un grupo humano con su ambiente específico, con el propósito fundamental de asegurar su supervivencia, poco conocido en la literatura antropológica: los salineros, grupo cuya subsistencia depende básicamente de la sal que artesanalmente sacan, en los meses de verano, de las lagunas costeras en la zona central (en este caso de la Laguna de Cahuil, ubicada en la desembocadura del Río Nilahue, provincia Cardenal Caro, VI Región).

Nuestra estada en la zona de Cahuil, en diversas ocasiones, nos permitió entender con bastante detalle el sistema cultural de los salineros tal como se daba en ese momento, principalmente en lo relacionado con el proceso de producción de la sal. El análisis de los resultados obtenidos en la oportunidad obligaba a presentar una serie de nuevas interrogantes que, en la práctica, significaban el inicio de investigaciones complementarias y la concurrencia de diversos especialistas para responderlas satisfactoriamente. Este trabajo pretende presentar dichas preguntas y bosquejar los caminos que eventualmente podemos usar para resolver nuestras inquietudes al respecto.

En nuestro país, la sal que consumimos proviene de minas de sal gema, de salares y de salinas de costa y toda ella tiene un elevado grado de pureza (DiAz GarcÉs, p. 48), (Vila, p. 359). El porcentaje de sal de costa en el total de la

*Este artículo proviene de un proyecto dirigido por Patricio Poblete, cuyo coinvestigador fue Daniel Quiroz. Recibió el financiamiento del Servicio de Desarrollo Científico, Artístico y de Cooperación Internacional de la Universidad de Chile, y contó con la cooperación de J.C. Olivares, P. Mege, E. Pino y M. Varas. 
producción anual de sal común ha experimentado variaciones significativas a lo largo de los años. Tenemos, por ejemplo, que en 1930 se produjeron 37.362 ton, de las que 6.078 (casi un 20\%) correspondió a sal de costa (Díaz Garcés, p. 49); en cambio, en 1950, de las 47.651 ton, sólo 4.542 (menos del 10\%) provenía de salinas de costa (VILA, p. 366). La producción anual de sal abastece ampliamente el mercado interno y, en ocasiones, se han exportado cantidades apreciables de este mineral (VILA, p. 359).

Las salinas se encuentran principalmente distribuidas entre las desembocaduras de los ríos Maipo y Mataquito. Entre estos puntos, merecen destacarse las Salinas de El Convento, en la desembocadura del Estero de Yali, las Salinas de Cahuil, en la desembocadura del Río Nilahue, las Salinas de Bucalemu, en la desembocadura del Estero Paredones, las Salinas de Boyeruca, en la desembocadura del Estero San Pedro de Alcántara, las Salinas de Llico, en las inmediaciones de la desembocadura del Río Mataquito. Podemos también encontrar salinas fuera de este territorio, tales como las Salinas de Pullalli, en la desembocadura del Estero La Ligua, las Salinas de Los Lobos y San Rafael, cerca de Quintero y las Salinas de El Tabo o Las Cruces, en los alrededores de Cartagena (Dłaz Garcés, pp. 52-53); (Pino SaAvedra, p. 78); (Vila, pp. 363-364). Nuestro interés se circunscribió, en definitiva, a las salinas situadas en la Provincia Cardenal Caro, VI Región.

\section{Características generales de la Producción de sal en las Salinas de Cahuil, Provincia Cardenal Caro, Sexta Región}

Podemos definir este sistema (adaptativo) señalando que en la Laguna de Cahuil, la sal se obtiene por la evaporación de agua de mar debido a la radiación solar, en estanques construidos ex profeso para tales efectos. La primera pregunta básica, entonces, que podemos plantearnos: ¿cuáles son las características globales más significativas del sistema de producción de la sal?

Las salinas son unidades productivas bastante complejas, estructuradas de manera muy armónica. Inmediatamente al costado de la laguna (fig. 9), encontramos el corral o corralón, estanque artificial donde se almacenan las aguas provenientes de la laguna, para ser posteriormente procesadas. Este estanque se encuentra separado de la laguna por un fuerte, muro de contención de barro y ramas, de $1,70 \mathrm{~m}$ de alto, $0,5 \mathrm{~m}$ de ancho y $50 \mathrm{~m}$ de largo, como promedio. Las aguas saladas de la laguna pasan al corral mediante una compuerta inserta en el fuerte, la que permite manipular, controlar y regular los flujos de agua salada. Entre la laguna y el corral existe sólo una gran compuerta, la que permite el paso rápido del agua. Contiguos al corral están los "sitios de salina", lugares donde se realiza efectivamente el proceso productivo. El agua es transportada del corral a los "sitios de salina" mediante compuertas más pequeñas. Un sitio de salina o abreviadamente sitio, está compuesto por calles de salina o simplemente calles, y las 


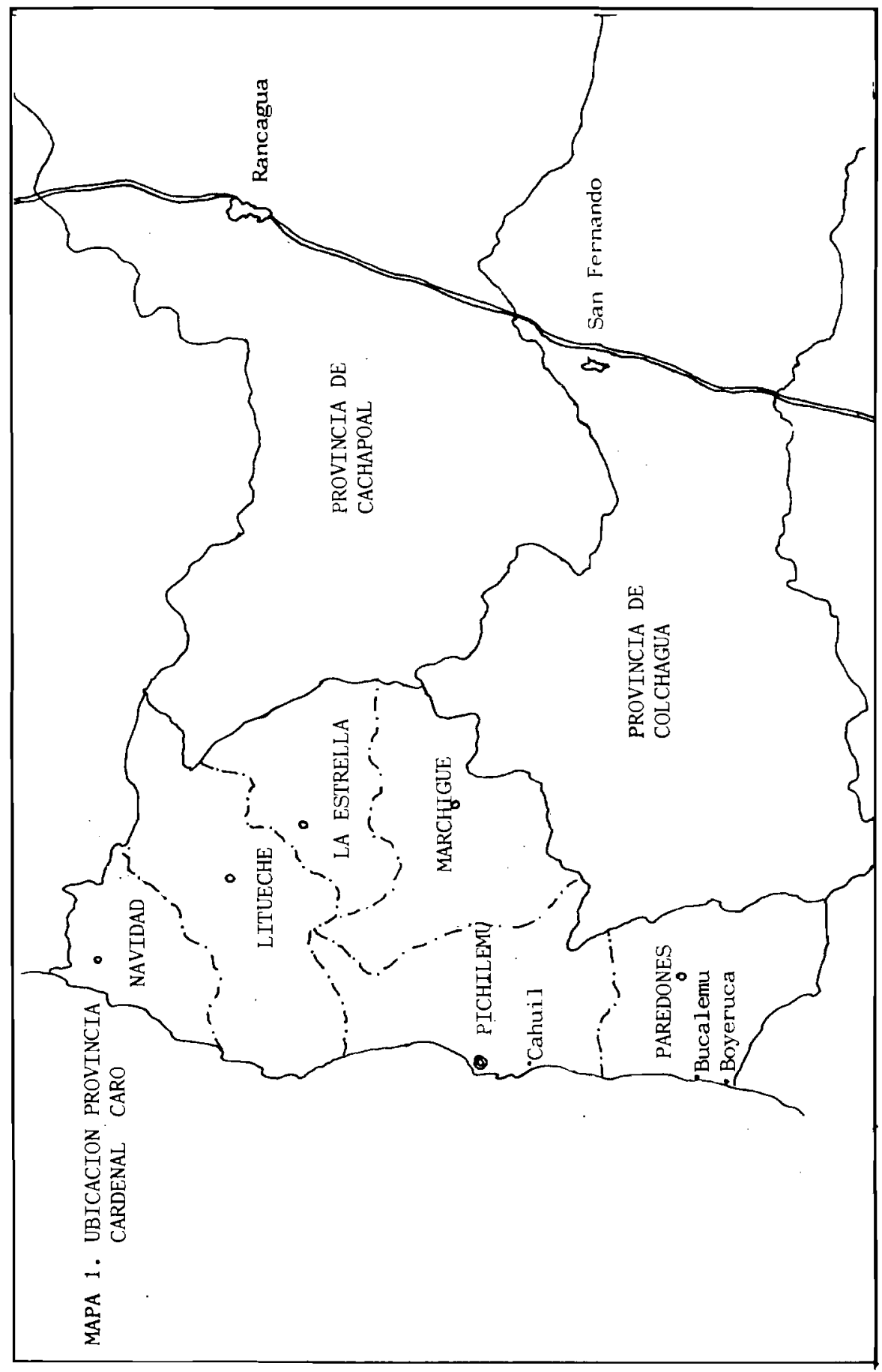




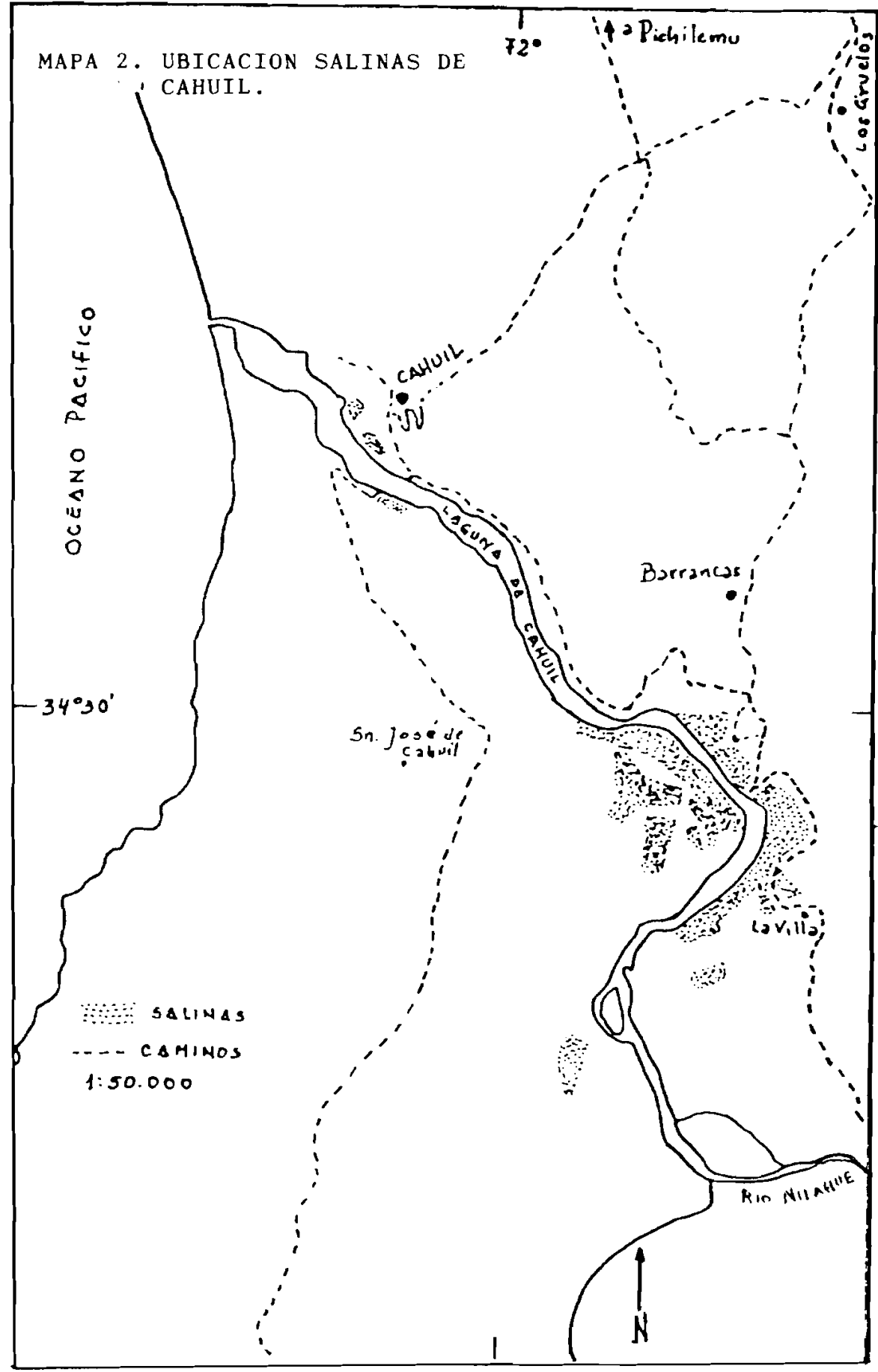


calles por piezas. Las piezas y las calles están separadas por pequeñas elevaciones de barro y ramas, denominadas parapetos, y conectados a través de un sistema de compuertas, canales y un sistema de vasos comunicantes. En cada calle es posible encontrar cuatro tipos diferentes de piezas, según el papel que les toca desarrollar en el proceso productivo. Las primeras piezas son las sancochadoras, siguen las cocedoras o cocederas, luego las recocedoras o recocederas y, finalmente, la cuajadora, llamada también cosechadora o cuartel. A través de este complejo sistema de estanques, esclusas y canales, es distribuida el agua salada de la laguna para obtener, después de unos 30 a 35 días, la cosecha de sal.

La producción de sal es la consecuencia de un ciclo que comienza con el desbarre, continúa con la llenada y termina con la salitrada o cosecha. El desbarre se inicia a fines de septiembre y termina a comienzos de noviembre. Comprende una serie de procesos diferentes: primero, la reparación del sistema de estanque, esclusas y canales; luego el desaguado, que consiste en sacar de las piezas el agua dulce que se estuvo acumulando durante los meses de invierno; más tarde se realiza el limpiado, que consiste en sacar el barro depositado en las piezas; finalmente, se efectúan el tostado, es decir el secado de cada uno de los estanques que conforman el sitio. La segunda parte del ciclo es la llenada, que implica el transporte del agua salada desde la laúna o laguna hasta el cuartel. El agua permanece durante un cierto tiempo en las diferentes piezas, en las sancochadoras, cocedoras, recocedoras, hasta que en el cuartel el agua cuaja, apareciendo en la superficie de la pieza los primeros granos de sal. Con este acontecimiento se da por iniciada la tercera etapa, la salitrada o cosecha que comienza a fines de diciembre, prolongándose hasta todo el mes de marzo. Son éstas las principales etapas del ciclo productivo de la sal.

En el proceso de producción de la sal, los salineros utilizan una variada gama de herramientas, las que casi en su totalidad manufacturan ellos mismos, lo que junto a su pertinente y adecuado manejo, representa estar en posesión de una tecnología adaptativa.

Dentro de estas herramientas, la pala (figs. 4 y 5 ) de madera, es tal vez la más importante, pues, en proporción al tamaño de las piezas, calles y sitios se convierte en una "mano" capaz de hacer las más variadas faenas: alular, rumbear (amontonar) y construir parapetos, entre otras.

Está construida sobre la base de una placa de madera aproximadamente de 30 por $40 \mathrm{~cm}$. El canto inferior de esta placa se aguza para permitir un mejor trabajo de la misma; luego se le agrega una ástil de $150 \mathrm{~cm}$ o más, de acuerdo a la estatura y comodidad del trabajador, un pequeño travesaño en el canto superior (en la unión de la placa y el astil) contribuye a hacerla más resistente. Esta herramienta es fundamental en la operación de desbarre y en el amontonamiento de la sal donde la agarrá (fig. 6), que depende de la habilidad del salinero para "palear" la sal, determinará parcialmente la calidad (de $1^{a}, 2^{a}$ o $3^{a}$ de acuerdo a su pureza) de la sal y por ende su precio.

Otra herramienta es el rastrillo (fig. 1), que sirve también tanto para amonto- 


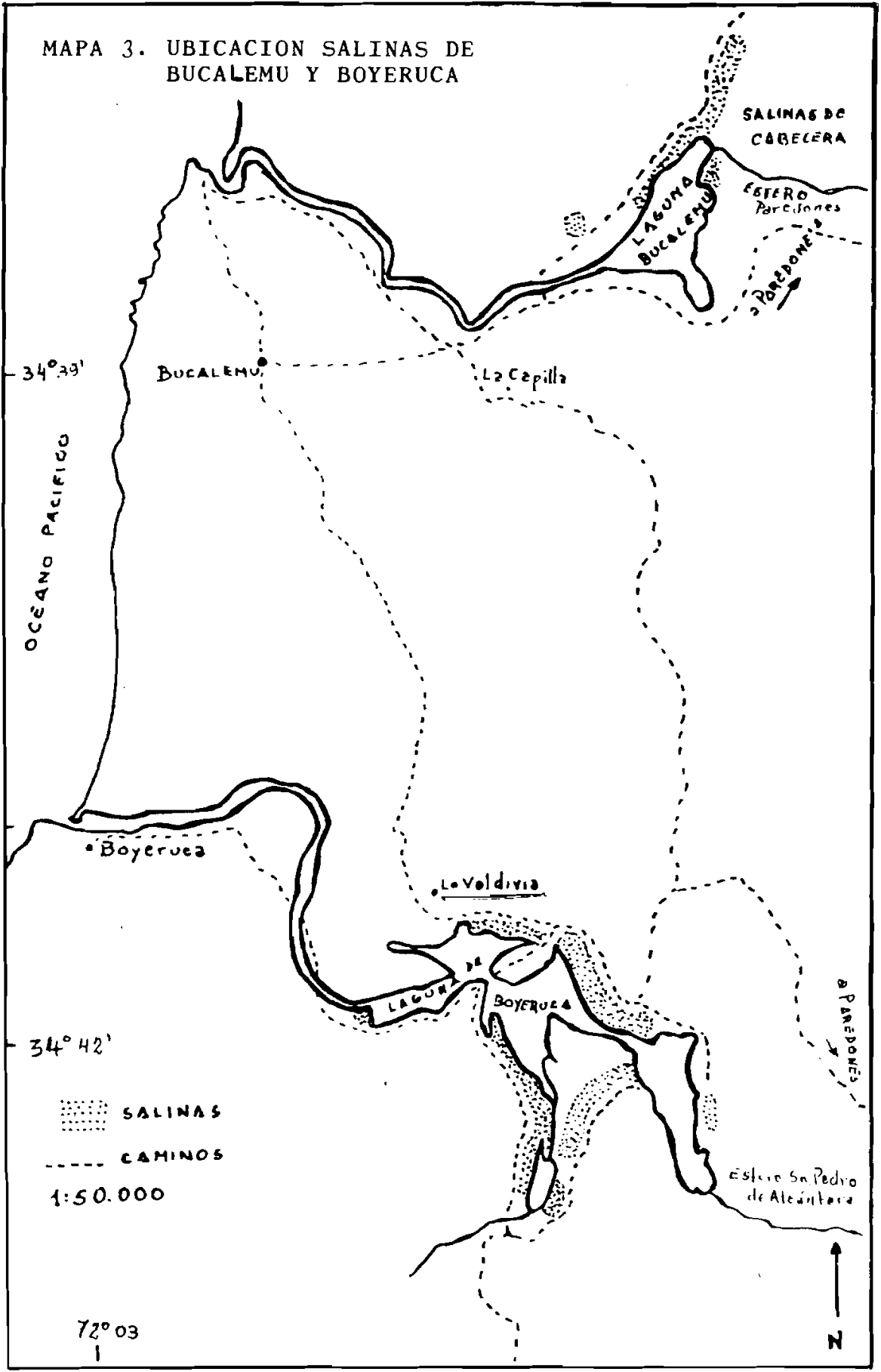




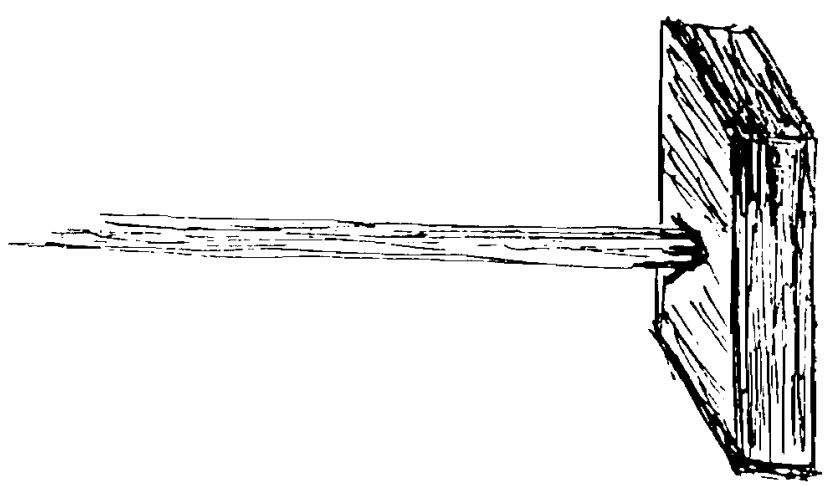

$\frac{5}{0}$
$\frac{1}{2}$
$m$
$\dot{b i}$
$\dot{m}$

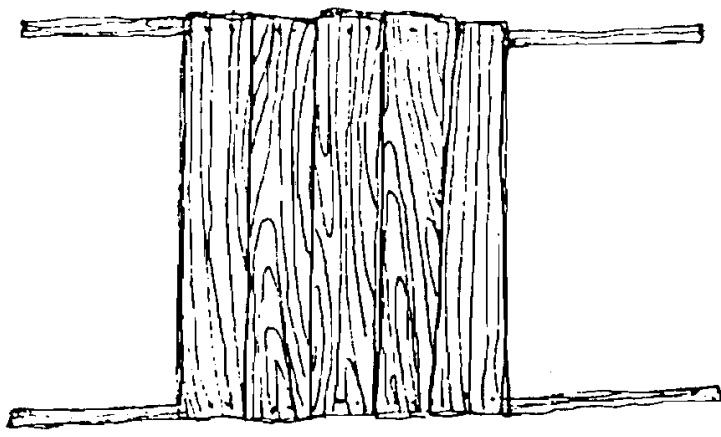

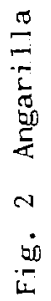

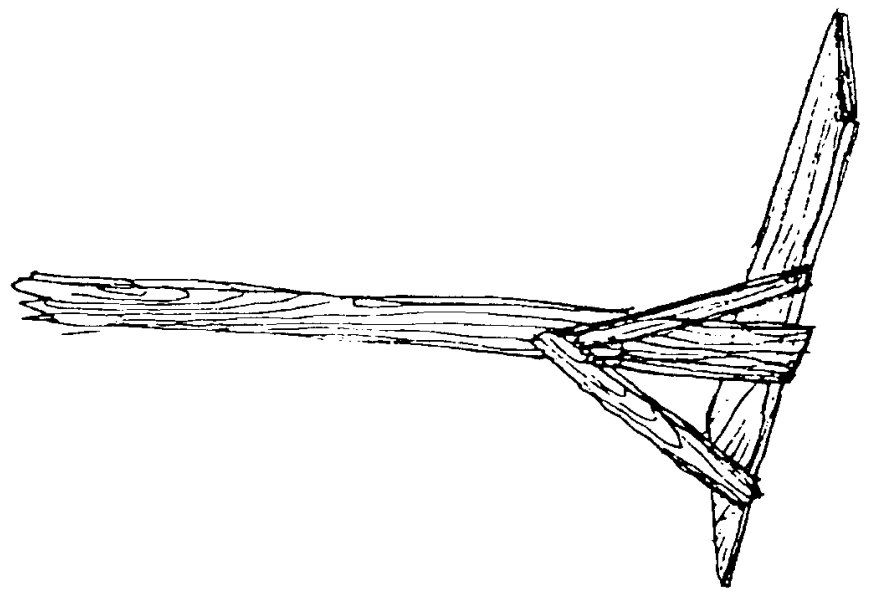

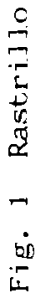


nar el barro (desbarre) como para reunir la sal. Al igual que la anterior, es construido de madera. Para ello se utiliza una tabla de $15 \mathrm{~cm}$ de ancho por $60 \mathrm{~cm}$ de largo; en la parte central del canto superior (transversalmente) se clava un listón de madera (huincha) de aproximadamente $5 \mathrm{~cm}$ de ancho y $150 \mathrm{~cm}$ de largo; éste se refuerza con dos tablillas colocadas diagonalmente desde el ástil hasta ambos extremos del mismo canto superior.

También encontramos aquí otra herramienta caracterizada por sus polifunciones: la angarilla (fig. 2) la que es utilizada en el transporte del barro (desbarre) y en el transporte de la sal (salitrada); está concebida para ser manipulada por dos personas; consta de una placa central, compuesta de varias tablas, que se une a ambos lados o largueros que sirven para asirla. Algunas tienen en los extremos de los largueros unos sacados, que permiten una mejor adaptación de la mano y evita que se resbale, así como también la comodidad de manipulación. El tablero central mide unos $70 \mathrm{~cm}$ de largo por $50 \mathrm{~cm}$ de ancho; los largueros se prolongan más de $50 \mathrm{~cm}$ en ambos sentidos del tablero.

El pisón (fig. 3) se utiliza para emparejar el piso de las piezas en una de las fases del desborre. De su adecuado uso depende que no se suelte dicho piso y no ensucie la sal. Esta función del apisonado algunas veces es complementada con el paso de un tubo de cemento hueco (20 a $25 \mathrm{~cm}$ de diámetro y $80 \mathrm{~cm}$ de largo) como rodillo. Para los efectos de la construcción del pisón se utiliza un trozo de madera de 40 por $40 \mathrm{~cm}$ de superficie y un espesor de aproximadamente $20 \mathrm{~cm}$; sobre él, en la parte central se efectúa un orificio en el cual se empotra un ástil. Al igual que en la construcción de la pala, sus dimensiones dependerán de la estatura y comodidad del trabajador (por lo general no alcanza los $150 \mathrm{~cm}$ ).

Por esto último aparece la herramienta que requiere mayor elaboración: el matiador (fig. 8), que consiste en una especie de recipiente (cubo de madera o lata) montada en un eje que se une a un cabestrante (de más de $200 \mathrm{~cm}$ de alto), el que, mediante articulaciones en base de alambres y listones y movido por una persona pendularmente, trasvasija el agua de una pieza a otra. Se ubica siempre en un sector de esquinas, donde se involucran varias piezas, pertenecientes a dos calles, inserto en el interior de un amplio, pero no profundo, pozo.

Pasemos ahora a mencionar las características del sistema en los otros lugares de salinas en la zona que nos ocupa.

\section{El sistema de los Salineros en la Provincia Cardenal Caro, Sexta Región}

Es importante aclarar, si nos preocupamos de establecer la extensión específica de un determinado sistema, las dimensiones concretas que él mismo posee. Cuando hablamos de los "salineros" de Cahuil nos estamos refiriendo a todos aquéllos que trabajan en las salinas situadas en ambas riberas de la Laguna de Cahuil: en la norte, Cahuil, Lo Angosto, Barrancas y Valle Grande; en la sur, La Huala, El Culén, La Isla, El Sauce y Crucecita. Cada uno de estos lugares se 


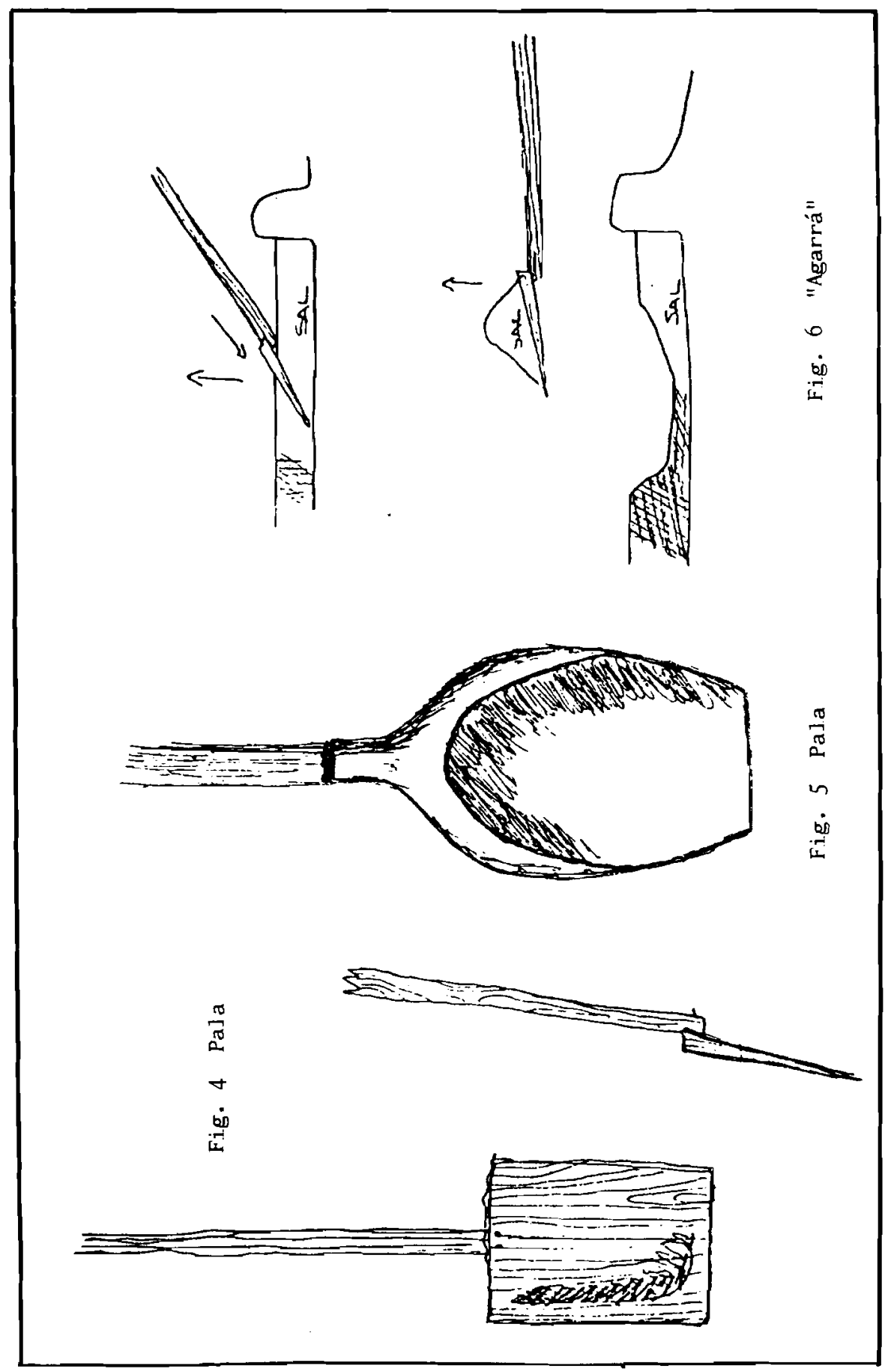




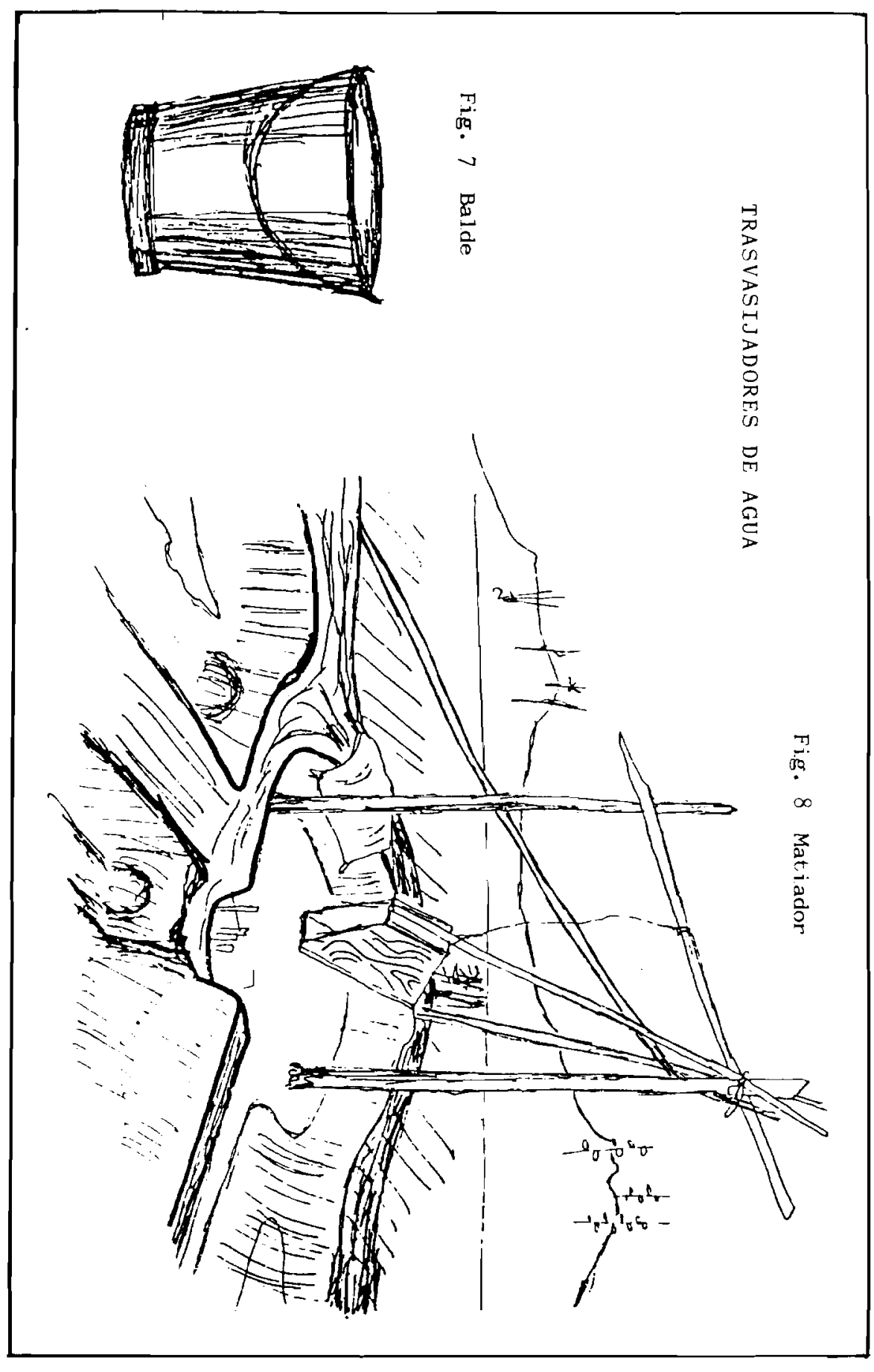

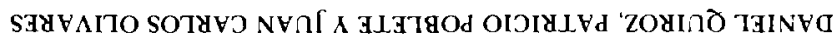


encuentra separado del otro por porciones de ribera sin salinas, distancia que va desde el 1/2 km hasta los 2 km (MANRíQUEZ, 1955, p. 34). En nuestra investigación estudiamos principalmente las salinas de Cahuil, Valle Grande o La Villa y Crucecita.

Luego de analizar los resultados obtenidos surgió la necesidad de contrastarlos con informaciones provenientes de otras realidades similares. Por ello se escogió estudiar, con el mismo detalle y temática, aquellas salinas existentes en la Provincia Cardenal Caro (VI Región). Según nuestros datos, las otras salinas que habian en la provincia estaban ubicadas en los márgenes de las lagunas de Bucalemu y Boyeruca, siendo las más importantes las Salinas de Cabeceras, en la Laguna de Bucalemu, y las Salinas de Lo Valdivia, en la Laguna de Boyeruca. Elegimos estas salinas teniendo presente, como en el caso de la Laguna de Cahuil, hay más de una localidad con salinas en los bordes de las lagunas.

Durante el transcurso de una de nuestras estadas en la zona, en octubre de 1981, comprobóse un hecho completamente inesperado: las salinas de Cabeceras habían dejado de explotarse desde hace varios años y, en todo el contorno de la Laguna de Bucalemu no habían salinas. En cambio, las salinas de Lo Valdivia estaban en plena producción, siendo una de las más importantes en la región. Estas salinas, ubicadas en la ribera norte de la Laguna de Boyeruca, en lo esencial, no se diferenciaban mayormente de las conocidas en la Laguna de Cahuil, tanto en sus aspectos sociales, económicos, legales y culturales. Podíamos, por tanto, generalizar los resultados obtenidos en el caso de las Salinas de Cahuil y aplicarlos a las salinas de la costa de la zona central de Chile. De esta manera, consideramos que podemos hablar del "modo adaptativo salinero", como un estilo de vida específico desarrollado por diversos grupos de personas en distintos lugares de similar característica.

El sistema que hemos descrito es relativamente uniforme para las salinas del país, tanto para las observadas por nosotros como para otras, de las cuales tenemos informaciones menos completas. El sistema (adaptativo) salinero representa un mecanismo que han desarrollado los habitantes de la costa para aprovechar una característica fisiográfica peculiar de su litoral, terrenos bajos ubicados en la desembocadura de esteros y pequeños ríos, en los cuales penetra el mar durante las altas mareas, permitiendo así la explotación humana de la sal.

\section{Los "Salineros" en México y Colombia: UNA COMPARACIÓN ILUSTRATIVA}

Nuestro interés iba un poco más allá de lo planteado. Queríamos saber si este sistema podíamos encontrarlo en otras regiones del mundo, especialmente en países económicamente similares al nuestro, tales como las naciones latinoamericanas. En la práctica, deseábamos extender el método comparativo hasta donde fuese posible. Sin embargo, es éste un trabajo largo que no puede completarse 


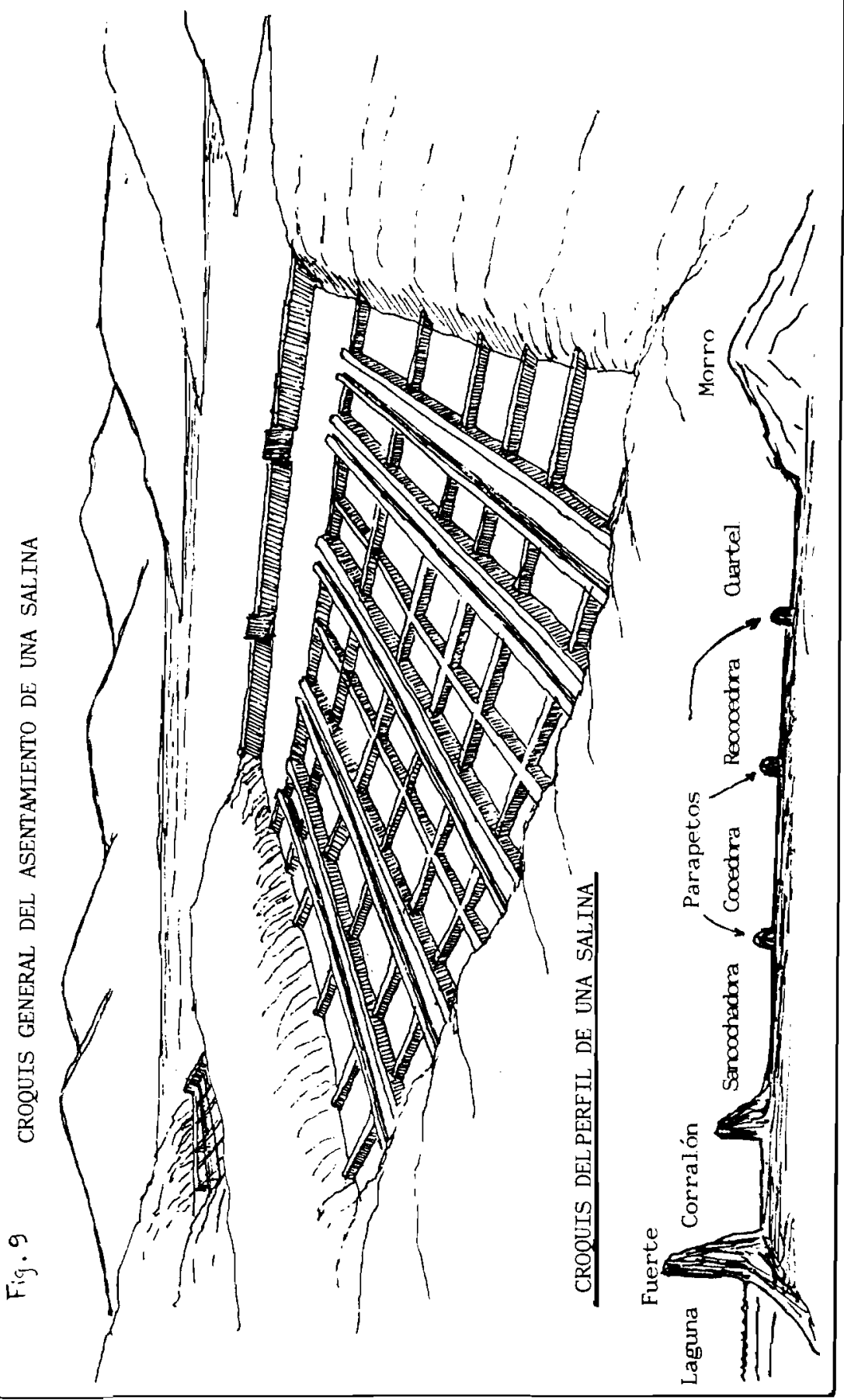


sin una revisión exhaustiva de una gran cantidad de literatura general, la que no nos asegura, tampoco, que encontremos lo que estamos buscando.

Como resultado de una búsqueda más o menos sistemática, encontramos un artículo que nos entrega valiosos datos sobre la presencia del mismo modo adaptativo en la Costa de Nayarit, México. En forma resumida, queremos entregar la descripción que hace J.R. Moriarty del sistema adaptativo de los Salineros de Las Cabras (Nayarit, México): "Los estanques y los diques internos sirven para ir separando la salmuera en las distintas etapas de evaporación y refinamiento de la sal. Los diques que dividen los estanques se hacen manualmente con el aluvión salino sedimentado en los estuarios, el cual se endurece al secar. Los estanques se utilizan en tres series: el primero para evaporación y concentración de la salmuera, el segundo intermedio o de "cal" y el tercero de cristalización o recolección de la sal. El sistema de canales entre los estanques sirve tanto para dirigir el agua de mar en la marea alta y mantenerla a un nivel constante, teniendo en cuenta la evaporación, como para drenar el concentrado de salmuera del estanque de evaporación al de "cal" y de éste al de cristalización. La sal se amontona en el centro de los diques internos de cristalización y se deja secar; de ahí se transporta a los procesos de refinamiento en las fábricas" (MORIARTY, p. 65). En términos generales no existen diferencias significativas con el sistema observado por nosotros en Cahuil y Lo Valdivia.

También encontramos antecedentes sobre la producción de sal de costa en la zona de La Guajira, Colombia, y tenemos datos sobre el tipo de explotación que ahí se efectúa (CARDALE dE SCHRimpFF, lámina 6). Al parecer en esta región no se encuentran los complejos sistemas de estanques, esclusas, canales y montículos, construidos por el hombre, sino más bien la utilización de grandes estanques donde se deposita, en forma natural, el agua de mar, durante los ciclos de marea alta, la que es evaporada por los fuertes rayos solares del tórrido verano tropical. Un buen ejemplo de este sistema, a una gran escala, lo constituyen las Salinas de Manaure, ubicadas en la Península de La Guajira, Colombia (BARING, pp. 129-132). Para Moriarty, este sistema u otro muy parecido, es el antecedente inmediato del observado en la zona de Las Cabras, Nayarit, México (p. 65). La construcción de estanques, esclusas y canales tiene por objeto mejorar la calidad y cantidad de la producción de sal:

"Las pozas de evaporación natural podían ser expandidas simplemente excavando para ampliar sus capacidades y la construcción de canales permitía el paso de un volumen superior de agua de mar en las pozas excavadas $/ . .1$. Esto condujo, indudablemente, a la elaboración de estanques artificiales y al desarrollo de prácticas simples de ingeniería, en la construcción de canales y diques. $\mathrm{El}$ experimento, la observación y el descubrimiento accidental, en períodos largos de tiempo, permite el desarrollo de modificaciones que llevan a aumentar la producción y a mejorar el producto" (MoriarTY, p. 67). 


\section{Los Salineros de Cahuil:}

\section{SU PASADO, DESARROLLO Y FUTURAS PROYECCIONES}

El sistema de explotación de las salinas en las costas de la zona central fue "inventado" durante una época específica en la historia. Nos es desconocido el momento de su ocurrencia, así como las causas que influyeron para que se produjera el cambio tecnologico señalado. No pretendemos precisar la fecha, pero sí nos interesa conocer, aproximadamente, la época en que se produjo. Por eso, quisimos rastrear la presencia del sistema adaptativo en el tiempo, tratando de descubrir indicios que nos permitieran pronunciarnos, de manera clara, sobre los problemas anteriormente planteados. Este trabajo debe ser, indudablemente, realizado con los métodos y técnicas de la historia, y, en lo posible, por historiadores. Aunque no contábamos con los especialistas ni tampoco dominábamos completamente la metodología histórica, pensamos que no debiamos renunciar a alcanzar esos conocimientos, así es que nos decidimos a dar el paso, aunque fuera en forma intuitiva.

De acuerdo a nuestros datos, durante todo el presente siglo, el sistema no ha sufrido modificaciones importantes, sobre todo en sus aspectos tecnológicos. Esta aseveración es confirmada por los datos que aparecen en trabajos relacionados con el tema, tales como los F. Manríquez (1955a, 1955b), sobre las mencionadas salinas de Cahuil, el de Y. Pino Saavedra (pp. 78-81), relacionado con el vocabulario usado por los salineros, y el de C. Díaz Garcés (pp. 52-53), sobre la industria de la sal en Chile. Un somero análisis de estas fuentes muestra que el sistema de explotación permaneció sin grandes variaciones durante el presente siglo, detectándose una disminución en la cantidad de salinas trabajadas efectivamente en la región comprendida entre los ríos Maipo y Mataquito, zona en la que se concentraba el mayor número de salinas de costa del país.

La referencia más temprana que tenemos sobre la existencia de salinas en la costa de la zona central nos la ofrece J. de Bibar, en su trabajo Crónica Y Relación Copiosa y Verdadera de las costas del Reino de Chile, escrita en 1558. En él nos dice que "hay muy buenas salinas de sal en la laguna que tengo dicha de Topocalma y en Quillota", y que además, "hay otras salinas y en otras muchas partes" (p. 133). Describe una de las salinas, ubicada en la llamada "Laguna de Topocalma", "de mil y cuatrocientos pasos de largo y media legua en redondo", "a dos tiros de piedra de la mar, la cual no entra dentro ni la agua de la laguna va a la mar por estar cercada de unos promontorios de tierra" (p. 75). Los "naturales" se proveían de sal en la laguna, durante los meses de verano, en "gran cantidad" (pp. 74-75). Finalmente, Bibar agrega que "a lo que entiendo, debe tener el asiento esta laguna en el asiento de lo salado del agua de la mar, a cuya causa se fragua aquella sal y cuaja en el asiento abajo porque, cuando la secan, sale como losas llanas de grueso de cuatro dedos y de una mano y más" (p. 75). La sal cosechada "es tan blanca como cristal, y en verano, como tiene poca 
agua la laguna, penetra más el sol su calor en la tierra, hace que la primera sal que sacan es rubia o roja, y la que sacan de abajo de ésta es más blanca" (p. 75).

Otra referencia importante a las salinas la encontramos en la HisTórica Relación del Reino de Chile, escrita por A. de Ovalle y publicada en 1646, donde se nos habla de las salinas de la llamada Laguna de Rapel. En esta laguna, "cerrándose la boca por donde se continúa con el mar, por el mes de enero, cuando son más fuertes los soles, se cuaja el agua que queda dentro, de manera que se hace una costra de más de dos y tres palmos de grueso de sal blanca y de muy buen sabor" (pp. 53-54). Señala Ovalle que no todos los años sucede esto, pero la gente, "de un año suelen proveerse para otros" (p. 54).

Es interesante destacar una información que entrega Ovalle sobre las salinas, de bastante utilidad para el problema de la cronología y desarrollo del sistema adaptativo. Para Ovalle, "nunca faltan por lo menos las salinas que se hacen a mano, que son como unas pequeñas lagunas en que, entrando el agua de invierno, se convierte en sal la que queda dentro y, como es menos la materia, surte el efecto con menos sol" (p. 54). Estas salinas "que se hacen a mano" pueden corresponder a primarios estanques artificiales construidos, como se explicita, para acelerar el proceso de evaporación del agua de mar, disminuyendo su profundidad. Por ello, planteamos que, por lo menos a principios del siglo xvir, ya se estaban formando las salinas de la costa de la zona central, tal como las conocemos hoy en día.

Ya avanzado el siglo xviII, en la obra Historia Geographica e Hidrographica con derrotero general correlativo al Plan del Reino de ChIle, escrita en 1760 a petición del entonces Gobernador de Chile Dn. Antonio de Amat y Junyent, aparecen una serie de datos de interés sobre el tema que nos preocupa, sobre todo referentes a la cantidad y distribución de las salinas de la zona central. Según este trabajo, tenemos que en la Hacienda de Santo Domingo, que se extendía entre el Río Maipo y el Estero Yali, había "una laguna pequeña donde cuaja sal", y en la hacienda de Bucalemu, que se extendía entre el Estero Yali y el Río Rapel, habían "salinas copiosas" (pp. 428-430). Esto, respecto de las salinas ubicadas entre los ríos Maipo y Rapel.

En relación a las salinas existentes entre los ríos Rapel y Mataquito la Historia Geographica... nombra a nuestras conocidas de Bucalemu y Cahuil, "dos lagunas, que cuajan sal" (p. 437) y de Boyeruca, "dos lagunas, que cuajan sal" (p. 439), agregándoles las de Vichuquén, "dos salinas que cuajan sal en los veranos" (p. 436). Sobre las Salinas de Vichuquén, que aún no han sido estudiadas, señala que el valle en el que corre el "riachuelo" de Vichuquén, está "poblado de mucha gente, que comercia en las salinas" (p. 433). Esto nos permite afirmar que, por lo menos a mediados del siglo XVIII, todas las salinas conocidas actualmente en el interfluvio Rapel-Mataquito, se encontraban en funcionamiento pleno.

A fines del siglo xix, E. Espinoza, en su Geografia Descriptiva de la RePúblicA DE ChILE, nos describe algunas características de las salinas existen- 
tes en los estuarios del interfluvio Rapel-Mataquito. Señala que, en la margen norte del estuario de Cahuil se encuentra un "pequeño caserío", con el mismo nombre, "cuyos pobladores esplotan las abundantes salinas del estuario, que aunque se venden a bajo precio en los pueblos del interior, forman, sin embargo, una fuente de recursos" (p. 273) y nos dice que en los estuarios de Bucalemu y Boyeruca, "se elabora una abundante producción de sal marina que surte gran parte de las provincias centrales i forma una fuente de recursos a sus esplotadores" (p. 281). Espinoza agrega que la "producción anual se estima en $\mathbf{2 4 0 . 0 0 0}$ quintales métricos de sal, que si hubiera medios fáciles de locomoción para el centro del país, daría provechosísimos resultados a los que esplotan esta producción" (p. 281).

Un interesante y completo panorama de las salinas a fines del siglo pasado se encuentra en el Diccionario Geográfico Postal de la República de Chile de F. Fuentes. Según este autor, además de las Salinas de Cahuil (que extrañamente no las nombra), entre los ríos Rapel y Mataquito existirían las Salinas de Bucalemu y Cabeceras en la Laguna de Bucalemu, las Salinas de Boyeruca y Lo Valdivia en la Laguna de Boyeruca, y las Salinas de California, Maravillas y Membrillo en las cercanías de Llico (p. 208), "a 10 kilómetros al N. del Puerto de Llico" (Risopatrón, p. 789). Es así como podemos plantear la existencia y permanencia de cuatro núcleos de salinas en el interfluvio Rapel-Mataquito a fines del siglo xıx: el primero, en las márgenes de la Laguna de Cahuil, el segundo, en los bordes de la Laguna de Bucalemu, el tercero, a orillas de la Laguna de Boyeruca, y un cuarto, en las inmediaciones de Llico.

Finalmente, ya en nuestro siglo, en un trabajo sobre la industria de la sal en Chile, C. Díaz Garcés señala que "las principales salinas de costa en actual producción y que se distinguen por su calidad, son: Cahuil, Pichilemu (debe decir Bucalemu), Bolleruca, Llico, El Tabo, El Convento, Pullalli y Quintero" (p. 52; hemos subrayado las salinas correspondientes al sector estudiado, las que coinciden con las determinadas para fines del siglo xix). Díaz Garcés agrega que el trabajo en las salinas se hace "recibiendo el agua de mar en un estanque, del que se manda por medio de un canal a las bateas de cristalización, donde el agua se evapora, debido a la acción solar y los vientos" (p. 53).

\section{Los Salineros: Sus antecedentes Y MANIFESTACIONES ARQUEOLÓGICAS}

Sobre este punto no es mucho lo que podemos señalar, ya que la arqueología local está solamente esbozada. Solamente disponemos de los trabajos de J.T. Medina, A. Oyarzún y J. Montané de un valor arqueológico sólo preliminar, pero sin ninguna relación con nuestro tema. De acuerdo, entonces, con los antecedentes etnohistóricos que hemos presentado anteriormente, podemos afirmar, tentativamente, que el sistema fue un rasgo hispánico que se asentó sobre una base de explotación indígena, de mucho menor magnitud. 


\section{Conclusiones}

Hemos querido mostrar los principales aspectos de un estilo de vida que conlleva varios modos de vida. Por lo expuesto, el que corresponde al "salinero" es el subordinante frente a otros subordinados complementarios: agricultor, pescador, jornalero, obrero (de aserradero), u otro. Así como también sólo hemos querido describir el ciclo productivo de la sal, sin referirnos a las etapas que constituyen los ciclos de distribución y de consumo, ciclos que cierran el circuito económico en la explotación de las salinas.

Un elemento importante que deberá ser investigado más a fondo, es el referente a la propiedad de las salinas y a las modalidades de su uso, arrendamiento, mediería, etc., así como el sistema organizacional de los trabajadores la presencia de trabajos individuales y colectivos, y de otros elementos, que en conjunto con los anteriores constituirían un claro sistema adaptativo.

Ahora bien, la sal tiene en un ciclo de producción que va desde las últimas semanas de septiembre a fines de marzo, lo que implica dejar alrededor de cinco meses y medio dedicados a otra actividad, la que por lo general se efectúa en la zona, primando la de salinero, actividad que se transmite de padres a hijos. Esto último y a pesar de que el factor económico lleva a algunos a buscar un trabajo "permanente" hace que esta actividad sea tan peculiar a través de varios siglos y que en términos generales tanto su tecnología como otros aspectos culturales no se hayan modificado mayormente.

Existe un aspecto que también necesita ser profundizado: el por qué ciertas salineras dejan de explotarse, en especial las de Cabecera, donde al parecer confluyeron varias causas, y si también son estas mismas causas, u otras, o una combinación de ellas, las que provocaron una situación similar en otras "ex salinas".

No obstante todo lo expuesto, creemos que el tema no se agota ahí, ni mucho menos, toda vez que un sistema adaptativo posee una dinámica especial y aunque no logremos visualizar sus modificaciones en un período corto, éstas se están produciendo y requieren de su conocimiento científico.

\section{Bibliografía}

Amat y Junyent, M. De. "Historia Geographica e Hidrographica con derrotero general correlativo al Plan de el Reyno de Chile". Revista Chilena de Historia y Geografia, $\mathrm{N}^{\circ} 55$, [Santiago], 1925-1926, pp. 425-458.

Baring, A. 1981. "Los guajiros". Pueblos de la Tierra, [Barcelona], Tomo v, Salvat, 1981, Pp. 126-135.

B1BAR, J. DE. Crónica y Relación copiosa y verdadera de los Reynos de Chile. Santiago, Fondo Histórico y Bibliográfico José Toribio Medina, 1966.

Cardale de SChrimpfF, M. Las Salinas de Zipaquira: su explotación indigena. Bogotá, Fundación de Investigaciones Arqueológicas Nacionales, 1981. 
Díaz Garcés, C. El Cloruro de Sodio: su industria en Chile: Santiago, Lagunas y Quevedo, 1933.

Espinoza, E. Jeografía Descriptiva de la Repriblica de Chile. Santiago, Barcelona, 1897.

Fuentes, F. 1899. Diccionario Jeografico-Postal de la Repriblica de Chile. Santiago, Universo, 1899.

Manriquez, F. Salinas de Cahuil. Santiago, Memoria de Prueba para optar al título de Profesor de Estado en Historia, Geografia y Educación Clvica, Universidad de Chile, $1955 \mathrm{a}$.

Manríquez, F. "Salinas de Cahuil". Informaciones Geográficas, vol. v, 1955 b, pp. 23-42.

Medina, J.T. Los restos indígenas de Pichilemu. Santiago, Elzeviriana, 1908.

Montane, J. "Elementos precerámicos de Cahuil". Notas del Museo Arqueológico de La Serena $N^{o} 8$. [La Serena], 1960, pp.

MOR1ARTY, J. "The influence of strand plain morphology on the development of primitive industries along the Costa de Nayarit, México". América Indígena, vol. xxv, $\mathrm{N}^{\circ} 1,1965$, pp. 65-77.

Ovalle, A. DE. Histórica Relación del Reyno de Chile. Santiago, Instituto de Literatura Chilena, 1969.

Oyarzún, A. "Crónica Pichilemu-Cahuil". Publicaciones del Museo de Etnologia y Antropologia de Chile, vol. I, Nos 4-5, 1917, pp. 297-300.

Pino, Y. "Anotaciones sobre vocablos y acepciones usadas en Chile". Anales de la Facullad de Filosofía y Educación. [Santiago] (Universidad de Chile), Sección Filologia, vol. I1, No 1 , 1937-1938, pp. 77-88.

Quiroz, D. y P. Poblete. Los Salineros de la Provincia Cardenal Caro, VI Región. m.s., 1982.

Risopatrón, L. Diccionario Jeográfico de Chile. Santiago, Universitaria, 1924.

VILA, T. Recursos minerales no-metalicos de Chile. Santiago, Universitaria, 1953. 